Pacific Journal of Mathematics

ON CONVEX HULLS OF TRANSLATES 


\section{ON CONVEX HULLS OF TRANSLATES}

\section{GLICKSBERG}

1. Let $G$ be a locally compact group, $g$ a closed subgroup, each taken with its left invariant Haar measure. For $f$ in $L_{1}(G)$ let $f^{s}$ denote a right translate of $f\left(f^{s}(x)=f(x s)\right)$ and let $C_{f}$ denote the closed convex hull of the set $\left\{f^{s}: s \in g\right\}$ of right translates of $f$ by elements of $g$.

Recently Reiter [9] considered the problem of determining the distance in $L_{1}$ from the origin to $C_{f}$ and proved, when $g$ is abelian ${ }^{1}$ and the homogeneous space $G / g$ of left cosets $x g$ has a left invariant measure, that the distance is given by the expression

$$
\int_{\theta / g}\left|\int_{\theta} f(x s) d s\right| d x^{\prime}
$$

where integration is with respect to the invariant measures (suitably normalized), and $x$ is an element of the coset $x^{\prime}$. Now this suggests the following (overly general) question: suppose one has a semigroup $S$ of operators of norm 1 on a Banach space $B$; under what sort of conditions can one explicitly determine the distance from the origin to the convex hull of the orbit $S x$ of an $x$ in $B$ ?

In the present note we give a simple approach to certain problems of this sort (Lemma 2.1), which yields some information whenever $S$, in the terminology of [3], is right amenable, and leads to an explicit determination of the distance in a variety of cases (see 2.2-2.4). In particular we obtain (in 3.2) a considerable strengthening of Reiter's result in which $g$ assumes the rôle of a very well behaved transformation group on a locally compact space $G$, while Haar measure on $G$ is replaced by an essentially $g$-invariant measure.

But for a recent extension by Day [4] of the Markov-Kakutani theorem [1, p. $114 ; 5$, p. 456] we should have to take our semigroup $S$ abelian; for the reader's convenience we shall begin by giving a (rather different) proof of Day's result.

2. Let $S$ be a semigroup, $m(S)$ the usual supremum normed space of all bounded complex functions on $S$. For $f \in m(S)$ let $f_{s}(t)=f(s t)$, $s, t \in S$. A left invariant mean $M$ on $m(S)$ is a nonnegative (hence continuous) linear functional for which ${ }^{2}$

$$
\langle 1, M\rangle=1,\left\langle f_{s}, M\right\rangle=\langle f, M\rangle
$$

Received February 20, 1962. Supported by the National Science Foundation through Grant G14779. The author is indebted to Dr. H. Reiter for several helpful comments.

1 Or somewhat more generally, the product of a compact and an abelian group.

2 Right invariant means are defined analogously, with $\left\langle f^{s}, M\right\rangle=\langle f, M\rangle$. 
for all $s \in S, f \in m(S)$.

Day's extension [4] of the Markov-Kakutani theorem can be stated as follows. ${ }^{3}$

Let $K$ be a compact convex subset of a locally convex topological vector space $X, S$ a semigroup of continuous affine maps of $K$ into itself for which $m(S)$ has a left invariant mean. Then $S$ has a common fixed point $x$ in $K: s x=x$, all $s$ in $S$.

(To see this we shall essentially apply our mean $M$ to the vector valued function $s \rightarrow s x$ ( $x$ some element of $K)$. In order to make this legitimate we shall convert $M$ into a measure on a "compactification" of $S$.)

Let $K^{K}$ be the space of all (not necessarily continuous) maps of $K$ into itself, taken in the topology of pointwise convergence. $K^{K}$ is compact by Tychonoff's theorem, as is the closure $\Sigma$ of $S \subset K^{K}$.

We can of course compose elements $\sigma$ and $\tau$ of $\Sigma$ and it is easily seen that $\Sigma$ is itself a semigroup; however for our purposes it is sufficient to note that $s \sigma \in \Sigma$ for $s \in S, \sigma \in \Sigma:$ if $s_{\delta} \rightarrow \sigma$ in $\Sigma, s_{\delta} \in S$, then $s_{\delta} x \rightarrow \sigma x$, all $x$ in $K$, whence $s s_{\delta} x \rightarrow s \sigma x, s$ being continuous, so $s \sigma=\lim s s_{\delta}$, and $s \sigma \in S=\Sigma$. And clearly the continuity of $s \in S$ implies $\sigma \rightarrow s \sigma$ is continuous from $\Sigma$ into itself.

Now let $M$ be our left invariant mean on $m(S)$; for $f$ a continuous function on $\Sigma$ we have the restriction $f \mid S$ in $m(S)$, so

$$
f \rightarrow\langle f \mid S, M\rangle
$$

is a nonnegative linear functional on the space $C(\Sigma)$ of all continuous functions, which assumes the value 1 at the constant function 1 . Consequently by the Riesz representation theorem we have a nonnegative measure $\mu$ of norm 1 on $\Sigma$ for which

$$
\langle f \mid S, M\rangle=\int f(\sigma) \mu(d \sigma) .
$$

Evidently for $s$ in $S, f_{s} \mid S=(f \mid S)_{s}$, so $(\sigma \rightarrow f(s \sigma)$ being continuous because $\sigma \rightarrow s \sigma$ is)

$$
\int f(s \sigma) \mu(d \sigma)=\left\langle f_{s} \mid S, M\right\rangle=\left\langle(f \mid S)_{s}, M\right\rangle=\langle f \mid S, M\rangle=\int f(\sigma) \mu(d \sigma) .
$$

Let $x \in K$, and consider the vector valued integral

3 Abelian semigroups have invariant means on $m(S)$ (a consequence of the MarkovKakutani theorem), so this is an extension; also solvable groups have such means; for other instances, see [3].

${ }_{4}$ That is, preserving convex combinations. 


$$
\int \sigma x \mu(d \sigma)
$$

which exists in the Riemann sense since $\sigma \rightarrow \sigma x$ is continuous, and so lies in $K$ by convexity. Since any $s$ in $S$ is affine and continuous,

$$
s \int \sigma x \mu(d \sigma)=\int s \sigma x \mu(d \sigma)
$$

while for any $x^{*}$ in $X^{*}$,

$$
\left\langle\int s \sigma x \mu(d \sigma), x^{*}\right\rangle=\int\left\langle s \sigma x, x^{*}\right\rangle \mu(d \sigma)=\int\left\langle\sigma x, x^{*}\right\rangle \mu(d \sigma)
$$

by (2.1), so

$$
\left\langle\int \operatorname{s\sigma x} \mu(d \sigma), x^{*}\right\rangle=\left\langle\int \sigma x \mu(d \sigma), x^{*}\right\rangle,
$$

and $s \int \sigma x \mu(d \sigma)=\int \sigma x \mu(d \sigma)$ by (2.3) and (2.4). Thus (2.2) is the desired fixed point. ${ }^{5}$

The following simple consequence of Day's result and the HahnBanach theorem forms the core of our approach to the problem raised in $\S 1$.

LEMMA 2.1. Let $S$ be a semigroup of operators of norm $\leqq 1$ on the normed space $B$, and suppose $m(S)$ has a right invariant mean. Let $C_{x}$ denote the closed convex hull of $\{s x: s \in S\}, x \in B$. Then the distance from the origin to $C_{x}$ in $B$, dist $\left(0, C_{x}\right)$, if positive, is given by

$$
\operatorname{dist}\left(0, C_{x}\right)=\left|\left\langle x, x^{*}\right\rangle\right|
$$

where $x^{*}$ is an element of norm 1 of the adjoint space $B^{*}$, fixed under the adjoint semigroup $S^{*}=\left\{s^{*}: s \in S\right\}$.

Of course 2.1 can only be applied to yield (2.5) explicitly when we can sufficiently limit the possible candidates for such an $x^{*}$. But as we shall see, this can be done in a variety of cases. (Note that 2.1 says nothing if $\|s\|<1$ for some $s$ in $S$, since the distance then is always zero; we have not insisted that the elements of $S$ are of norm 1 simply to avoid needless verifications in our applications.)

${ }^{5}$ It may be worth noting that what we have needed, rather than a left invariant mean on all $m(S)$, is a left invariant mean $M$ on the subspace spanned by 1 and functions of the form $t \rightarrow\left\langle s t x, x^{*}\right\rangle$ and their conjugates. (For then $M$ has a nonnegative extension to all of $C(\Sigma)$, invariant on the appropriate subspace.) 
To proceed to the proof, let $c=\operatorname{dist}\left(0, C_{x}\right)>0$. As a consequence ${ }^{\sigma}$ of the Hahn-Banach theorem there is an element $y^{*}$ of $B^{*}$ of norm 1 for which

$$
c \leqq\left|\left\langle y, y^{*}\right\rangle\right|, \quad y \text { in } C_{x} .
$$

Let $C_{y^{*}}^{*}$ be the weak* closed convex hull of $\left\{s^{*} y^{*}: s\right.$ in $\left.S\right\}$, endowed with the weak* topology; since $C_{y^{*}}^{*}$ is clearly contained in the unit ball of $B^{*}$, $C_{y^{*}}^{*}$ is compact. And for any $x^{*}$ in $C_{y^{*}}^{*}$,

$$
c \leqq\left|\left\langle y, x^{*}\right\rangle\right|, \quad y \text { in } C_{x}
$$

indeed it suffices to verify (2.7) for the dense set of $x^{*}$ of the form $\sum_{1}^{N} c_{n} s_{n}^{*} y^{*}, c_{n} \geqq 0, \Sigma c_{n}=1$, and for $y$ in $C_{x}$

$$
c \leqq\left|\left\langle\sum_{1}^{N} c_{n} s_{n} y, y^{*}\right\rangle\right|=\left|\left\langle y, \sum_{1}^{N} c_{n} s_{n}^{*} y^{*}\right\rangle\right|
$$

by (2.6), since $\sum_{1}^{N} c_{n} s_{n} y \in C_{x}$. Note that by (2.7) and the definition of $c$, $\left\|x^{*}\right\| \geqq 1$, hence $\left\|x^{*}\right\|=1$, for every $x^{*}$ in $C_{y^{*}}^{*}$.

Now $S^{*}$ provides a semigroup of continuous affine maps of $C_{y^{*}}^{*}$ into itself; and since $s \rightarrow s^{*}$ is an anti-isomorphism of $S$ onto $S^{*}$, our right invariant mean on $m(S)$ clearly induces a left invariant mean on $m\left(S^{*}\right)$. Thus Day's theorem applies and we have an $x^{*}$ in $C_{y^{*}}^{*}$ fixed under $S^{*}: s^{*} x^{*}=x^{*}, s$ in $S$. This is of course the desired functional, and it only remains to prove (2.5).

But $x^{*}$ is constant on $C_{x}$ since

$$
\left\langle\sum_{1}^{N} c_{n} s_{n} x, x^{*}\right\rangle=\left\langle x, \sum_{1}^{N} c_{n} s_{n}^{*} x^{*}\right\rangle=\left\langle x, \sum_{1}^{N} c_{n} x^{*}\right\rangle=\left\langle x, x^{*}\right\rangle
$$

for $c_{n} \geqq 0, \Sigma c_{n}=1, s_{n}$ in $S$. So by $(2.7), c \leqq\left|\left\langle x, x^{*}\right\rangle\right|=\left|\left\langle y, x^{*}\right\rangle\right| \leqq$ $\|y\|\left\|x^{*}\right\|=\|y\|$ for all $y$ in $C_{x}$, and (2.5) follows since $\|y\|$ can be chosen arbitrarily close to $c=\operatorname{dist}\left(0, C_{x}\right)$.

An obvious case in which 2.1 leads to explicit determination of the distance is that in which $S$ is also "ergodic," in the sense that the fixed points of $S^{*}$ form a one-dimensional subspace of $B^{*}$, spanned say by the unit vector $x_{0}^{*}$. For the $x^{*}$ of 2.1 is then a unimodular multiple of $x_{0}^{*}$, whence

$$
\operatorname{dist}\left(0, C_{x}\right)=\left|\left\langle x, x_{0}^{*}\right\rangle\right|
$$

${ }^{6}$ This is best known in the real case (and is a special case of the Eidelheit separation. theorem [2, p. 22]). One obtains it as follows. If $B_{0}$ is the open unit ball in $B$ then $c B_{0}-C_{x}$ has interior $(c>0$ !) while 0 is not in the interior, so by (a form of) the HahnBanach theorem $[1$, p. $71 ; 2]$ there is a real linear functional $F$ on $B$. $\leqq 0$ on $c B_{0}-C_{x}$. Normalizing $F$ so $\|F\|=1, F\left(c B_{0}\right)$ is the interval $(-c, c)$, and $F\left(C_{x}\right) \geqq c$; so setting $H(x)=$ $F(x)-i F(i x), x \in B$, yields as usual a complex linear $H$ of norm 1 , with $\left|H\left(C_{x}\right)\right| \geqq \operatorname{Re} H\left(C_{x}\right)=$ $F\left(C_{x}\right) \geqq c$. 
if the distance is positive, while

$$
\begin{aligned}
\left|\left\langle x, x_{0}^{*}\right\rangle\right| & =\left|\left\langle x, \Sigma c_{n} s_{n}^{*} x_{0}^{*}\right\rangle\right|=\left|\left\langle\Sigma c_{n} s_{n} x, x_{0}^{*}\right\rangle\right| \\
& \leqq\left\|\Sigma c_{n} s_{n} x\right\|
\end{aligned}
$$

shows the same is true if the distance is zero.

An explicit example of this sort is the first of the following three, which illustrate the application of 2.1 .

2.2. Let $(X, \mathscr{B}, \mu)$ be a (totally) $\sigma$-finite measure space (so the adjoint of $L_{1}(\mu)$ is $L_{\infty}(\mu)$ ). Let $S$ be a semigroup of $(1-1)$ maps of $X$ onto itself which, along with their inverses, are measurable and measure preserving. Suppose $S$ is $\mu$-ergodic (that is, if $E \nabla F$ denotes the symmetric difference of $E$ and $F$ in $\mathscr{B}, \mu\left(s^{-1} E \nabla E\right)=0$ for all $s$ in $S$ implies $\mu(E)=0$ or $\mu(X \backslash E)=0)$, while $m(S)$ has a left invariant mean. ${ }^{7}$ For $f \in L_{1}(\mu)$, let $C_{f}$ be the closed convex hull of $\{f \circ s: s$ in $S\}$. Then

$$
\operatorname{dist}\left(0, C_{f}\right)=\left|\int_{X} f(x) \mu(d x)\right|
$$

Here our left invariant mean on $m(S)$ translates into a right invariant mean for the (anti-isomorphic) semigroup of isometric maps $f \rightarrow f \circ s$, while our assumption that $S$ is $\mu$-ergodic implies (as is well known) that the only fixed points of the adjoint semigroup are the constants, and so (2.8) follows.

Of course when $\mu$ is finite one might obtain (2.8) from general mean ergodic theorems, but this is not the case in general, where $C_{f}$ need not contain an $S$-invariant element (as in the example of footnote 7 ).

2.3. Our next example is far less trivial. Let $G$ be a locally compact abelian group, $\mu$ a nonnegative regular Borel measure on $G$, of norm 1 (a probability measure). Let $g$ be the smallest closed subgroup of $G$ which carries $\mu$, and let $T$ be the operator on $L_{1}(G)$ sending $f \rightarrow \mu * f$, $S=\left\{T^{n}: n \geqq 1\right\}$. Then if $C_{f}$ is the closed convex hull of $\left\{T^{n} f: n \geqq 1\right\}$,

$$
\operatorname{dist}\left(0, C_{f}\right)=\int_{\theta / g}\left|\int_{g} f(x s) d s\right| d x^{\prime},
$$

where the measures are the appropriate Haar measures, normalized so that

$$
\int_{\theta} f(x) d x=\int_{\sigma / g} \int_{g} f(x s) d s d x^{\prime}
$$

$x^{\prime}$ being the coset $x g$ of $x$.

${ }^{7}$ For example, with our measure space the usual one formed from the positive reals (Lebesgue measure), one could take $S=\left\{s^{n}: n \geqq 1\right\}$, where $s$ is the ergodic transformation of the half line given on p. 84 of $[6]$. 
First note that whenever $s \rightarrow f(x s)$ is integrable on $g$ (i.e., for almost all $\left.x^{\prime}\right), \int_{g} T^{n} f(x s) d s=\int_{g} f(x s) d s$ since $\mu$ is carried by $g$. Thus if $\sum c_{n}=1$,

$$
\begin{aligned}
& \int_{G / g}\left|\int_{g} f(x s) d s\right| d x^{\prime}=\int_{\theta / g}\left|\int_{g} \sum c_{n} T^{n} f(x s) d s\right| d x^{\prime} \\
& \leqq \int_{G / g} \int_{g}\left|\sum c_{n} T^{n} f(x s)\right| d s d x^{\prime}=\left\|\sum c_{n} T^{n} f\right\|_{1},
\end{aligned}
$$

by (2.10), so (2.9) clearly holds if $\operatorname{dist}\left(0, C_{f}\right)=0$, and we can henceforth assume the distance is positive. Thus 2.1 clearly applies: we have a $\varphi$ of norm 1 in $L_{\infty}(G)$ for which

$$
\operatorname{dist}\left(0, C_{f}\right)=\left|\int_{G} f(x) \varphi(x) d x\right|=\left|\int_{G / g} \int_{g} f(x s) \varphi(x s) d s d x^{\prime}\right|
$$

while $T^{*} \varphi=\varphi$. Now the set of $\varphi$ in $L_{\infty}(G)$ satisfying $T^{*} \varphi=\varphi$ is precisely the subspace $E$ orthogonal to the range of $f \rightarrow f-\mu * f=f-T f$, and we want to see these functions $\varphi$ are constant on cosets $\bmod g$.

But the closure of the range of this map, $I=\left\{f-\mu * f: f \in L_{1}(G)\right\}^{-}$, is a closed ideal in $L_{1}(G)$, whose hull clearly is the set of characters $\hat{g}$ in the dual group $G^{\wedge}$ for which $1=\hat{\mu}(\hat{g})\left(=\int_{G} \hat{g}(x) \mu(d x)\right)$. Since $\mu \geqq 0$ and of norm 1 , these characters are just those identically 1 on the carrier of $\mu$, hence just those in $g^{\perp}$, the group of characters identically 1 on $g$. Since [7] $g^{\perp}$ is a set of spectral synthesis, we know $E$, the subspace of $L_{\infty}(G)$ orthogonal to $I$, is the weak* closed span of the characters in $g^{\perp}$; hence ${ }^{7 a} E$ consists of functions constant on cosets mod $g$, as desired, and, in particular our $\varphi$ in (2.12) is such a function.

Consequently (since $\|\varphi\|_{\infty}=1$ ) by (2.12)

$$
\operatorname{dist}\left(0, C_{f}\right) \leqq \int_{\epsilon / \sigma}\left|\int_{g} f(x s) d s\right| d x^{\prime} ;
$$

since the reverse inequality was obtained in (2.11), (2.9) follows.

Note that we could just as well take a family of probability measures $\left\{\mu_{\alpha}\right\}, S$ the semigroup generated by all the corresponding maps $f \rightarrow \mu_{\alpha} * f$, and $g$ the smallest closed subgroup of $G$ carrying all our measures. For $I$, in the above argument, would replaced by the closure of the set of all finite sums $\Sigma\left(f_{n}-\mu_{\alpha_{n}} * f_{n}\right)$, an ideal whose hull is the set of characters identically 1 on all carriers, hence with hull $g^{\perp}$ as before.

Finally one could take $\mu$ to be any measure of norm 1 , but then one sees (quite easily) that if $\operatorname{dist}\left(0, C_{f}\right)>0$ for some $f, \mu$ has to be the product of a character and a probability measure; for 2.1 provides a nonzero $\varphi$ orthogonal to $I$, so that $I$ is a proper ideal and must have a nonvoid hull. If $\hat{g}$ lies in the hull, so $\hat{\mu}(\hat{g})=\int \hat{g}(x) \mu(d x)=1$ then

7a That $\psi^{s}=\psi$ in $L_{\infty}, s \in g, \psi \in E$, is immediate; that the assertion follows is a result of Reiter (Ann. Math., to appear; see 3.1 below). 
$\nu=\hat{g} \mu \geqq 0$. (The corresponding result for such a measure is of course easily derived from the original case since $\hat{g}(\mu * f)=(\hat{g} \mu) *(\widehat{g} f)$.)

2.4. Our next example is a variant of 2.3 , although $G$ could be nonabelian (if we take care in writing products). $G$ will again be a locally compact abelian group and $\mu$ a probability measure on $G$, but now $T: f \rightarrow \mu * f$ will act on $C_{0}(G)$, the continuous functions vanishing at infinity on $G$. Let $g$ again be the smallest closed subgroup of $G$ carrying $\mu$. With $S$ and $C_{f}$ as before we can now assert that $\operatorname{dist}\left(0, C_{f}\right)=$ 0 for all $f$ in $C_{0}(G)$ unless $g$ is compact; and if $g$ is compact

$$
\operatorname{dist}\left(0, C_{f}\right)=\sup _{x}\left|\int_{g} f(x s) d s\right| \text {. }
$$

Suppose the distance is positive for some $f$, so by 2.1 we have a $\nu$ in $C_{0}(G)^{*}=M(G)$ (= finite complex regular Borel measures on $G$ ) of norm 1 for which $T^{*} \nu=\nu$, and

$$
\operatorname{dist}\left(0, C_{f}\right)=\left|\int_{\theta} f(x) \nu(d x)\right| \text {. }
$$

Since $f * \mu(y)=\int f\left(x^{-1} y\right) \mu(d x), T * \nu=\nu$ becomes $\mu^{\prime} * \nu=\nu$, where $\mu^{\prime}(E)=$ $\mu\left(E^{-1}\right)$. As a consequence we conclude (as is well known) that $\delta_{x} * \nu=\nu$ for each $x$ in the carrier of $\mu^{\prime}$, where $\delta_{x}$ is the unit point mass at $x$. (Indeed, consider $K=\left\{\lambda \in M(G):\|\lambda\| \leqq 1, \mu^{\prime} * \lambda=\lambda\right\}$, a translation invariant weak* compact convex set. For an extreme point $\lambda$ of $K$, $\lambda=\mu^{\prime} * \lambda$ can be rewritten in terms of a weak ${ }^{*}$ convergent integral:

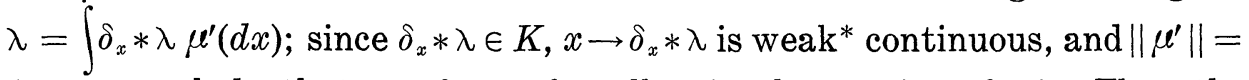
1 , we conclude that $\lambda=\delta_{x} * \lambda$, for all $x$ in the carrier of $\mu^{\prime}$. Thus the same is true for all $\lambda$ in $K$.)

Consequently we have $\delta_{x} * \nu=\nu$ for all $x$ in $g$, and $g$ must be compact; for if $\left\{x_{\gamma}\right\}$ is a net tending to infinity we clearly have $\left\|\delta_{x_{\gamma}} * \nu-\nu\right\| \rightarrow$ $2\|\nu\|=2$, and so this cannot hold for $x_{\gamma}$ in $g$.

Now let $\widetilde{C}_{f}$ be the closed convex hull of $\left\{f^{s}: s\right.$ in $\left.g\right\}$ in $C_{0}(G)$, so $C_{f} \subset \widetilde{C}_{f}$, and let $\mu_{g}$ be normalized Haar measure on $g$. Then $f * \mu_{g}$ is an element of least norm in $\widetilde{C}_{f}:\left\|\Sigma c_{n} f^{s_{n}}\right\| \geqq\left\|\Sigma c_{n} f^{s_{n}} * \mu_{g}\right\|_{\infty}=\left\|\Sigma c_{n} f * \mu_{g}\right\|_{\infty}=$ $\left\|f * \mu_{g}\right\|_{\infty}$, for $c_{n} \geqq 0, \Sigma c_{n}=1$, and $s_{n}$ in $g$ of course. Thus since our $\nu$ satisfies $\nu * \mu_{g}=\nu$,

$$
\begin{aligned}
\operatorname{dist}\left(0, C_{f}\right) \geqq \operatorname{dist}\left(0, \widetilde{C}_{f}\right) & =|| f * \mu_{g} \|_{\infty} \geqq\left|\left\langle f * \mu_{g}, \nu\right\rangle\right| \\
& =|\langle f, \nu\rangle|=\operatorname{dist}\left(0, C_{f}\right),
\end{aligned}
$$

and we obtain (2.13) since $\left\|f * \mu_{g}\right\|_{\infty}$ is its right side. ${ }^{8}$

8 This example could also be obtained from the mean ergodic theorem, which applies (since $f$ in $C_{0}(G)$ is weakly almost periodic) to yield an $h$ in $C_{f}$ of minimal norm. (Just as in the case of our $\nu$ above, one sees $h^{s}=h$ for $s$ in $g$, whence $g$ is compact if $h \neq 0$; since $h$ is constant on cosets $\bmod g$, $\|h\|_{\infty}=\sup _{x}\left|\int_{g} h(x s) d s\right|$, which clearly coincides with the right side of (2.13)) 
2.5. Finally we should note an alternative formulation of 2.1 which, although not useful for our purposes, has some interest: the assertion of 2.1 is equivalent to the assertion that, if $\operatorname{dist}\left(0, C_{x}\right)$ is positive, then

$$
\operatorname{dist}\left(0, C_{x}\right)=\operatorname{dist}(0, x+\operatorname{span}\{y-s y: y \text { in } B, s \text { in } S\}) ;
$$

(hence that the origin is equidistant from $C_{x}$ and a certain linear variety through it).

Indeed let $L$ denote the closed subspace formed from $\operatorname{span}\{y-s y: y$ in $B, s$ in $S\}$; then $\Sigma c_{n} s_{n} x=x+\Sigma c_{n}\left(s_{n} x-x\right)$ shows $C_{x} \subset x+L$, so

$$
\operatorname{dist}\left(0, C_{x}\right) \geqq \operatorname{dist}(0, x+L) \text {. }
$$

But the $x^{*}$ of 2.1 clearly has the constant value $\left\langle x, x^{*}\right\rangle$ on $x+L$, and since it has norm $1, \operatorname{dist}(0, x+L) \geqq\left|\left\langle x, x^{*}\right\rangle\right|=\operatorname{dist}\left(0, C_{x}\right)$. Thus the assertion of 2.1 implies that given in (2.14).

Conversely (2.14) implies the assertion of 2.1. For if we let $x^{*}$ be any element of $B^{*}$ of norm 1 vanishing on $L$ (and such will exist if $\left.\operatorname{dist}\left(0, C_{x}\right)=\operatorname{dist}(0, x+L)>0\right)$ then $s^{*} x^{*}=x^{*}$ follows, and so $x^{*}$ has the constant value $\left\langle x, x^{*}\right\rangle$ on $C_{x}$. Consequently one obtains $\operatorname{dist}\left(0, C_{x}\right)=$ $\left|\left\langle x, x^{*}\right\rangle\right|$ exactly as in the proof of 2.1 .

Note that (2.15) shows we do not need to assume our distance positive in (2.14). Moreover (2.14) shows that in explicitly computing $\operatorname{dist}\left(0, C_{x}\right)$ in the situation given in 2.1 , we are actually computing the norm in a quotient space of $B$; this was in fact the origin of Reiter's work.

3. We now turn to our version of Reiter's result, which has a generality due mainly to recent results of Świerczkowski [10]. Several remarks will be necessary before we state our result.

Let $X$ be a locally compact space, on which the locally compact group $g$ acts (on the right) as a transformation group, so that $(x, s) \rightarrow x s$ is continuous from $X \times g \rightarrow X$, and the identity of $g$ is the identity map. Let $X / g$ denote the corresponding orbit space, that is, the space of orbits $x g$ in the finest topology rendering the canonical map $\rho: X \rightarrow X / g$ continuous. We shall assume

(3.01) $X / g$ is locally compact (Hausdorff), and $\rho: X \rightarrow X / g$ is open, and, for any compact $K \subset X$

(3.02) $\{s \in g: K \cap K s \neq \phi\}$ is compact $g$ : alternatively,

$\left(3.02^{\prime}\right)\{(x, s): x \in K, x s \in K\}$ is compact in $X \times g$.

For example, we might take our locally compact $X$ to be (metrizable and) the simply connected covering space of a connected locally simply 
connected space $Y=X / g, g$ the fundamental group of ${ }^{9} Y$. As a simpler example take $X=G$ a locally compact group, $g$ a closed subgroup, so $X / g$ is the homogeneous space $G / g$, and (3.02) is evident. Or take $X=$ $Y \times g, Y$ an arbitrary locally compact space, and let $g$ act in the obvious (trivial) fashion.

Let $L(X)$ denote the vector space of (complex) continuous functions with compact support on $X$. Condition (3.02) insures that for any $f$ in $L(X), s \rightarrow f(x s)$ is in $L(g)$, and so we may form

$$
f^{\prime}\left(x^{\prime}\right)=\int_{g} f(x s) d s,
$$

where $d s$ is the element of left invariant Haar measure on $g$, and $x^{\prime}$ is the orbit $x g$ of $x$. (Left invariance of course implies (3.1) is independent of the particular $x$ in $x^{\prime}$.) Since, by (3.01), $\rho: X \rightarrow X / g$ is open the resulting function $f^{\prime}$ is continuous on $X / g$, hence lies in $L(X / g)$. Moreover by (3.01) every compact subset $K$ of $X / g$ is the image of some compact subset of $X$ (simply choose for each $x^{\prime}$ in $K$ some preimage $x$ and a compact neighborhood $V_{x}$; finitely many of the (compact) neighborhoods $\rho\left(V_{x}\right)$ cover $\left.K\right)$. As a consequence $f \rightarrow f^{\prime}$ maps $L(X)$ onto $L(X / g)$, exactly as in the case in which $X=G$ is a group, $g$ a closed subgroup $[11$, p. 43].

Let $\nu$ be any Radon measure on $X / g$, that is, a nonnegative regular Borel measure, finite on compacta. Since $f \rightarrow \int_{X / g} f^{\prime}\left(x^{\prime}\right) \nu\left(d x^{\prime}\right)$ is a nonnegative functional on $L(X)$, we have a corresponding unique Radon measure $\mu$ on $X$ (by the Riesz representation theorem):

$$
\int_{X} f(x) \mu(d x)=\int_{x / g} \int_{g} f(x s) d s \nu\left(d x^{\prime}\right),
$$

$f$ in $L(X)$. (Note that $\mu$ also uniquely determines $\nu$ since $L(X)$ maps onto $L(X / g)$.)

In [10, Th. 1] Świerczkowski has characterized the measures $\mu$ which so arise when $X=G$ is a group, $g$ a closed subgroup, and precisely the same proof applies in our more general setting. (One needs only the fact that, for $f, h$ in $L(X),(x, s) \rightarrow f(x) h(x s)$ is in $L(X \times g)$, which follows from $\left(3.02^{\prime}\right)$.) The induced measures $\mu$ are just those which translate (as one would expect from (3.2)) as follows, where ${ }^{10} \int f(x) \mu^{s}(d x)=$ $\int f^{s}(x) \mu(d x)$, and $\delta$ is the modular function [11] of $g$ :

${ }^{9}$ Here the set in (3.02) is finite, a consequence of the fact that $g$ acts freely $(x s=x t$ for some $x$ implies $s=t$ ). For suppose $\left\{s_{n}\right\}$ is a sequence of distinct elements of the set, so $k_{n} s_{n} \in K, k_{n} \in K$, all $n$. Passing to a subsequence we can assume $k_{n} \rightarrow k, k_{n} s_{n} \rightarrow k^{\prime}$, and all $k_{n}$ lie in a neighborhood $V$ of $k$ for which $\rho \mid V$ is a homeomorphism. Then $\rho\left(k_{n}\right)=$ $\rho\left(k_{n} s_{n}\right) \rightarrow \rho\left(k^{\prime}\right)$ and to $\rho(k)$, so $k^{\prime}=k s, s \in g$. So $k_{n} s_{n} s^{-1} \rightarrow k, k_{n} s_{n} s^{-1} \in V$ for $n \geqq n_{0}$, whence $k_{n} s_{n} s^{-1}=k_{n}, n \geqq n_{0}$, and $s_{n}=s, n \geqq n_{0}$, our contradiction.

10 Here $f^{s}(x)=f(x s)$ as earlier. Since Świerczkowski writes $f^{s}(x)=f\left(x s^{-1}\right)$, both (3 3) and (3.4) below feature $\delta(s)$ in place of $\delta\left(s^{-1}\right)$ in [10]. 


$$
\mu^{s}=\delta\left(s^{-1}\right) \mu
$$

in particular when $g$ is unimodular $(\delta \equiv 1)$ these are the $g$-invariant measures on $X$.

Now fix $\mu$ and $\nu$ satisfying (3.2). Usual monotoneity arguments show (as in [10]) that $f \rightarrow f^{\prime}$ extends to map the nonnegative (extended real valued) Baire functions on $X$ onto the corresponding set of functions on $X / g$ (since $L(X)$ maps onto $L(X / g)$ ), while (3.2) continues to hold for such functions; by (3.2) those nonnegative Baire functions which are $\mu$-integrable map into $\nu$-integrable functions of the same $L_{1}$ norm, so $f \rightarrow f^{\prime}$ extends to map $L_{1}^{+}(\mu)$, the nonnegative elements of $L_{1}(\mu)$, onto $L_{1}^{+}(\nu)$, hence to a map of $L_{1}(\mu)$ onto $L_{1}(\nu)$. Since (3.2) holds for all $f$ in $L_{1}^{+}(\mu)$, it continues to hold for $f$ in $L_{1}(\mu)$.

We shall require one further result of Świerczkowski [10, Th. 3], which extends to our setting without change: ${ }^{11}$ the kernel of the map $f \rightarrow f^{\prime}$ of $L_{1}(\mu)$ onto $L_{1}(\nu)$ is the closed span in $L_{1}(\mu)$ of the elements

$$
f^{s}-\delta\left(s^{-1}\right) f, \quad f \in L_{1}(\mu), \quad s \in g .
$$

(Note that by (3.3), $L_{1}(\mu)$ is $g$-invariant, so $f^{s} \in L_{1}(\mu)$.) This has the following consequence, which has been noted by Reiter.

Lemma 3.1. Let $\mu$ and $\nu$ satisfy (3.2), and suppose $\psi \in L_{\infty}(\mu)$ satisfies $\psi^{s}=\psi\left(\right.$ in $\left.L_{\infty}(\mu)\right)$ for all s in $g$. Then $\psi=\psi^{\prime} \circ \rho$ for some $\psi^{\prime}$ in $L_{\infty}(\nu)$ of (essential supremum) norm bounded by that of $\psi$.

Let $\langle\cdot, \cdot\rangle$ denote the pairing between $L_{1}$ and $L_{\infty}$, so $\langle f, \varphi\rangle=$ $\int_{X} f(x) \varphi(x) \mu(d x)$ for $f \in L_{1}(\mu), \varphi \in L_{\infty}(\mu)$. Then

$$
\begin{aligned}
\left\langle f, \varphi^{s}\right\rangle & =\int_{X} f(x) \varphi(x s) \mu(d x)=\int_{X / g} \int_{g} f\left(x s_{1}\right) \varphi\left(x s_{1} s\right) d s_{1} \nu\left(d x^{\prime}\right) \\
& =\int_{X / g} \int_{g} \delta\left(s^{-1}\right) f\left(x s_{1} s^{-1}\right) \varphi\left(x s_{1}\right) d s_{1} \nu\left(d x^{\prime}\right) \\
& =\int_{X} \delta\left(s^{-1}\right) f\left(x s^{-1}\right) \varphi(x) \mu(d x)=\left\langle\delta\left(s^{-1}\right) f^{s^{-1}}, \varphi\right\rangle
\end{aligned}
$$

so

$$
\left\langle f, \varphi^{s}\right\rangle=\left\langle\delta\left(s^{-1}\right) f^{s^{-1}}, \varphi\right\rangle, \quad f \in L_{1}(\mu), \quad \varphi \in L_{\infty}(\mu) .
$$

For our element $\psi$ in 3.1, this of course implies $\psi$ is orthogonal to $f^{s^{-1}}-\delta(s) f$, and so to the functions (3.4), hence orthogonal to the kernel of our map $f \rightarrow f^{\prime}$. Let $E$ denote the subspace of $L_{\infty}(\mu)$ orthogonal to the kernel, so $\psi \in E$.

11 [10] deals only with real $L_{1}$ spaces, but extension of this result to the complex case is trivial. 
Since $f \rightarrow f^{\prime}$ maps $L_{1}(\mu)$ onto $L_{1}(\nu)$, the adjoint map, which clearly sends $\psi^{\prime} \rightarrow \psi^{\prime} \circ \rho\left(\psi^{\prime} \in L_{\infty}(\nu)\right)$ by (3.2), must map $L_{\infty}(\nu)$ onto $E$. Consequently there is a $\psi^{\prime}$ in $L_{\infty}(\nu)$ for which $\psi=\psi^{\prime} \circ \rho$. Now

$$
\left\|\psi^{\prime}\right\|_{\infty}-\varepsilon<\left|\int_{X / g} h^{\prime}\left(x^{\prime}\right) \psi^{\prime}\left(x^{\prime}\right) \nu\left(d x^{\prime}\right)\right|=\left|\left\langle h^{\prime}, \psi^{\prime}\right\rangle\right|
$$

where $h^{\prime}$ is a positive multiple of an appropriate characteristic function, and of norm 1 in $L_{1}(\nu)$. Since $L_{1}^{+}(\mu)$ maps onto $L_{1}^{+}(\nu)$ under $f \rightarrow f^{\prime}, h^{\prime}$ is the image of a nonnegative $h$ in $L_{1}(\mu)$, and by (3.2) $\|h\|_{1}=\left\|h^{\prime}\right\|_{1}=1$. But $\psi^{\prime}$ maps onto $\psi$ under the adjoint map, so $\langle h, \psi\rangle=\left\langle h^{\prime}, \psi^{\prime}\right\rangle$, and thus

$$
\left\|\psi^{\prime}\right\|_{\infty}-\varepsilon\left\langle\left|\left\langle h^{\prime}, \psi^{\prime}\right\rangle\right|=|\langle h, \psi\rangle| \leqq\|h\|_{1}\|\psi\|_{\infty}=\|\psi\|_{\infty},\right.
$$

completing the proof of 3.1 .

We can now formulate our version of Reiter's result.

THEOREM 3.2. Suppose the locally compact space $X$ and the locally compact group $g$ acting on $X$ satisfy (3.01) and (3.02), and $\mu$ is a Radon measure on $X$ satisfying (3.3); let $\nu$ be the corresponding measure on $X / g$ satisfying (3.2).

Suppose g contains a subsemigroup $S$ which generates $g$ (topologically) for which there is a left invariant mean on the space $m(S)$ of bounded functions $^{12}$ on $S$. Then for every $f$ in $L_{1}(\mu)$ we have

$$
\inf \int_{X}\left|\sum_{1}^{N} c_{n} \delta\left(s_{n}^{-1}\right) f\left(x s_{n}^{-1}\right)\right| \mu(d x)=\int_{X / g}\left|\int_{g} f(x s) d s\right| \nu\left(d s^{\prime}\right),
$$

where the infimum is taken over all finite sums with $c_{n} \geqq 0, \Sigma c_{n}=1$, and $s_{n}$ in $S$.

Furthermore (3.6) continues to hold if the $s_{n}$ are allowed to range over any large subset of $g$, or the $c_{n}$ are allowed to be complex numbers summing to 1 , or both.

Slightly more can be claimed in the purely group setting $(X=G \supset g)$ if our measures are the (left invariant) Haar measure on $G$ and a relatively invariant measure on $G / g$ (see 4.1). (3.6) resembles Reiter's result most closely when $g$ is unimodular, but even asserts more, for example, when we take $X$ to be the group $R$ of additive reals, $g=S$ the subgroup of integers, where 3.2 takes the following form. Let $\mu$ be a periodic Radon measure, say of period 1, on $R$ (so $\mu(E+1)=\mu(E)$ for all Borel $E \subset R$ ); the corresponding $\nu$ is the restriction of $\mu$ to $[0,1)$,

12 Or just on the uniformly continuous functions; for we (in effect) apply our mean only to functions of the form $t \rightarrow\left\langle h, \phi^{s t}\right\rangle$ (cf. footnote 4 ), $h \in L_{1}(\mu), \phi \in L_{\infty}(\mu)$, and those are in fact uniformly continuous, a consequence of 3.3 below. 
viewed as the quotient group, so (3.6) asserts that for $f$ in $L_{1}(\mu)$,

$$
\inf \int_{-\infty}^{\infty}\left|\sum_{-N}^{N} c_{n} f(x+n)\right| \mu(d x)=\int_{0}^{1}\left|\sum_{-\infty}^{\infty} f(x+n)\right| \mu(d x) .
$$

Reiter's result yields the same assertion for Lebesgue measure, hence (almost immediately) for absolutely continuous $\mu$.

While it will be apparent that the hypothesized invariant mean is essential to our proof, we have no example showing it is essential to the result.

To proceed to the proof of 3.2 let $C_{f}$ be the closed convex hull of $\left\{\delta\left(s^{-1}\right) f^{s^{-1}}: s\right.$ in $\left.S\right\}$ in $L_{1}(\mu)$. We have to prove that $c=\operatorname{dist}\left(0, C_{f}\right)$ is precisely the right side of (3.6). From $\Sigma c_{n}=1$ we have (as in [9])

$$
\begin{aligned}
\int_{X / g}\left|\int_{g} f(x s) d s\right| \nu\left(d x^{\prime}\right) & =\int_{X / g}\left|\int_{g} \Sigma c_{n} \delta\left(s_{n}^{-1}\right) f\left(x s s_{n}^{-1}\right) d s\right| \nu\left(d x^{\prime}\right) \\
& \leqq \int_{X / g} \int_{g}\left|\Sigma c_{n} \delta\left(s_{n}^{-1}\right) f\left(x s s_{n}^{-1}\right)\right| d s \nu\left(d x^{\prime}\right) \\
& =\int_{X}\left|\Sigma c_{n} \delta\left(s_{n}^{-1}\right) f\left(x s_{n}^{-1}\right)\right| \mu(d x)
\end{aligned}
$$

by (3.2), so $c \geqq \int_{X / g}\left|\int_{0} f(x s) d s\right| \nu\left(d x^{\prime}\right)$, and it remains to prove $\mathrm{p}^{13}$

$$
c \leqq \int_{X / g}\left|\int_{g} f(x s) d s\right| \nu\left(d x^{\prime}\right) ;
$$

since this is obvious for $c=0$, we can assume $c>0$, and so apply 2.1.

Indeed, if $R_{s}$ is the operator on $L_{1}(\mu)$ defined by $R_{s} h=\delta\left(s^{-1}\right) h^{s^{-1}}$ then $R_{s}^{*} \varphi=\varphi^{s}$ since, by (3.5), $\left\langle R_{s} h, \varphi\right\rangle=\left\langle h, \varphi^{s}\right\rangle$; since $\left\|R_{s}^{*}\right\|=1,\left\|R_{s}\right\|=1$ (in fact $R_{s}$ is an isometry, as is easily seen). Now $s \rightarrow R_{s}$ is an antihomomorphism, since $R_{s} R_{t} h(x)=\delta\left(s^{-1}\right) R_{t} h\left(x s^{-1}\right)=\delta\left(s^{-1}\right) \delta\left(t^{-1}\right) h\left(x s^{-1} t^{-1}\right)=$ $R_{t s} h(x)$, so $\left\{R_{s}: s\right.$ in $\left.S\right\}$ is a semigroup having a right invariant mean on its bounded functions, and Lemma 2.1 applies: we have a $\varphi$ of norm 1 in $L_{\infty}(\mu)$ satisfying

$$
c=|\langle f, \varphi\rangle|
$$

for which $\varphi^{s}=\varphi$ in $L_{\infty}(\mu)$, for all $s$ in $S$.

Consequently $\varphi^{s}=\varphi$ for all $s$ in the dense subgroup $S$ generates algebraically in $g$. Now suppose that (as we shall see in Lemma 3.3 below) $s \rightarrow \varphi^{s}$ is a weak ${ }^{*}$ continuous map from $g$ into $L_{\infty}(\mu)$, so that $\varphi^{s}=\varphi$ in $L_{\infty}(\mu)$ for all $s$ in $g$. By Lemma 3.1 we then have $\varphi=\varphi^{\prime} \circ \rho$ where $\varphi^{\prime}$ is an element of $L_{\infty}(\nu)$ of norm $\leqq 1$, so by (3.9)

${ }^{13}$ Since (3.8) and (3.7) imply (3.6), there still remains the final assertion of 3.2 , but this follows trivially. (For the proposed changes in (3.6) can only decrease the left side, while by (3.7) no actual decrease is possible; this is precisely the argument used by Reiter to show [9] contained its precursor [8].) 


$$
\begin{aligned}
c & =|\langle f, \varphi\rangle|=\left|\int_{X / g} \int_{g} f(x s) \varphi(x s) d s \nu\left(d x^{\prime}\right)\right| \\
& =\left|\int_{X / g} \int_{g} f(x s) \varphi^{\prime}(\rho(x s)) d s \nu\left(d x^{\prime}\right)\right| \\
& =\left|\int_{X / g}\left(\int_{g} f(x s) d s\right) \varphi^{\prime}\left(x^{\prime}\right) \nu\left(d x^{\prime}\right)\right| \\
& \leqq \int_{X / g}\left|\int_{\sigma} f(x s) d s\right| \nu\left(d x^{\prime}\right)
\end{aligned}
$$

since $\varphi^{\prime}$ is of norm $\leqq 1$, which yields (3.8), and so (3.6).

Hence to complete the proof of 3.2 we have only to prove

LEMMA 3.3. Let $\mu$ satisfy (3.3). Then for $f$ in $L_{1}(\mu), s \rightarrow f^{s}$ is strongly continuous from $g$ into $L_{1}(\mu)$; consequently for $\varphi$ in $L_{\infty}(\mu)$, $s \rightarrow \varphi^{s}$ is weak* continuous (by (3.5) and the continuity of $\delta$ ).

Let $V$ be a fixed compact neighborhood of the identity in $g$. Since $\int_{X}\left|f^{s}(x)\right| \mu(d x)=\int_{X}|f(x)| \mu^{s}(d x)=\delta\left(s^{-1}\right)\|f\|_{1}$ by (3.3), the translation operators $f \rightarrow f^{s}$ on $L_{1}(\mu)$ corresponding to the elements $s$ of $V$ are uniformly bounded; thus it suffices to shows $s \rightarrow f^{s}$ is strongly continuous at the identity of $g$ for a dense subset of $L_{1}(\mu)$, in particular for $f$ in $L(X)$.

So suppose $f$ in $L(X)$ vanishes off the compact set $K \subset X$ and $\nu$ is the Radon measure on $X / g$ satisfying (3.2). Then for $t \in V$ by (3.2)

$$
\left\|f^{t}-f\right\|_{1}=\int_{X / g} \int_{g}|f(x s t)-f(x s)| d s \nu\left(d x^{\prime}\right) .
$$

Now the inner integral vanishes for $x^{\prime} \notin \rho(K)$, so we need only compute it for $x$ in $K$; and for $x$ in $K$ the integrand vanishes unless $x s t$ or $x s$ is in $K$. By (3.02') we have a compact $K_{1} \subset g$ for which $x s$ in $K$ and $x$ in $K$ imply $s \in K_{1}$, so our integrand vanishes (for $x$ in $K$ ) unless $s t$ or $s$ is in $K_{1}$, hence certainly if $s \notin K_{2}=K_{1} V^{-1} \cup K_{1} \supset K_{1} t^{-1} \cup K_{1}$. Consequently the inner integral is bounded by

$$
c_{t}=\sup \left\{|f(x s t)-f(x s)|: x \in K, s \in K_{2}\right\}
$$

multiplied by the Haar measure of $K_{2}$, for $x^{\prime}$ in $\rho(K)$. Since it vanishes for $x^{\prime} \notin \rho(K)$, and the compact set $\rho(K)$ has finite $\nu$ measure, (3.10) itself is bounded by $c \cdot c_{t}$, where $c$ is a constant independent of $t$. But $c_{t}$ clearly tends to 0 as $t$ tends to the identity of $g$, so our proof is complete.

When $g$ is abelian (so invariant means exist) one can easily see directly that the left side of (3.6) is the same whether the $s_{n}$ range (1) over $g$ or (2) only over a generating subsemigroup ${ }^{14} S$. Indeed by 3.3 it suffices to compute the left side of (3.6) using only a dense subset of our $s_{n}$. Thus in case (1) we can approximate the left side of (3.6) by

\footnotetext{
${ }^{14} \mathrm{I}$ am indebted to $\mathrm{K}$. deLeeuw for pointing this out to me.
} 


$$
\left\|\sum_{1}^{N} c_{n} f^{s-1} n_{n}\right\|_{1}
$$

where $s_{n}, t_{n} \in S$, since $S^{-1} S$ is dense in $g$. But since $g$ is unimodular $\left\|h^{s}\right\|_{1}=\|h\|_{1}, h$ in $L_{1}(\mu)$, so (3.11) coincides with $\left\|\sum_{1}^{N} c_{n} f^{s} s^{-1} t_{n} s\right\|_{1}$, where we may take $s=\prod_{1}^{N} s_{n}$, so that $s_{n}^{-1} t_{n} s \in S$ for all $n$. Hence the left side of (3.6) in case (1) is no less than in case (2), and since it could only be smaller, both are equal. But note that this is not at all apparent in the non-abelian case (and may of course, be false without some restriction).

4. Now let $G$ be a locally compact group, $g$ a closed subgroup, and suppose the homogeneous space $G / g$ of left cosets $x g=x^{\prime}$ carries a relatively invariant measure (which we shall denote by $d x^{\prime}$ ), i.e., for each $y$ in $G$ there is a $\chi(y)>0$ for which, for $f$ in $L(G / g)$,

$$
\int_{a / g} f\left(y^{-1} x^{\prime}\right) d x^{\prime}=\chi(y) \int_{a / g} f\left(x^{\prime}\right) d x^{\prime} .
$$

Then [11, pp. 42-45] $\chi$ is a continuous homomorphism of $G$ into the multiplicative positive reals, with

$$
\chi(s) \Delta(s)=\delta(s), \quad s \text { in } g,
$$

where $\Delta$ is the modular function of $G$; in fact the existence of a homomorphism $\chi$ satisfying (4.2) insures the existence of a relatively invariant measure satisfying (4.1). (In particular such a measure exists if $g$ is unimodular, e.g., with $\chi=\Delta^{-1}$.) With an appropriate normalization $[11$, p. 45],

$$
\int_{G} f(x) \chi(x) d x=\int_{G / g} \int_{g} f(x s) d s d x^{\prime},
$$

for all $f$ in $L(G)$, where $d x$ denotes the left invariant Haar measure on $G$. Consequently the measures $\chi(x) d x$ and $d x^{\prime}$ can assume the roles of $\mu$ and $\nu$, in which case (3.6) takes the form

$$
\inf \int_{\theta}\left|\Sigma c_{n} \delta\left(s_{n}^{-1}\right) f\left(x s_{n}^{-1}\right)\right| \chi(x) d x=\int_{G / g}\left|\int_{g} f(x s) d s\right| d x^{\prime},
$$

for $f$ in $L_{1}(\chi(x) d x)$. But now some simplification in (4.4) can be made by rephrasing it in terms of the element $f_{0}=f \chi$ of $L_{1}(G)$, and utilizing the involution $f \rightarrow f^{*}\left(f^{*}(x)=\Delta\left(x^{-1}\right) \overline{f\left(x^{-1}\right)}\right)$ available in $L_{1}(G)$.

COROLlARY 4.1. Let $G / g$ carry a relatively invariant measure satisfying (4.3). Suppose $g$ contains a generating subsemigroup $S$ as in 3.2. Then for every $f$ in $L_{1}(G)$

$$
\inf \int_{\theta}\left|\sum_{1}^{N} c_{n} f\left(s_{n} x\right)\right| d x=\int_{G / g}\left|\int_{g} f^{*}(x s) \chi(x s)^{-1} d s\right| d x^{\prime},
$$


and, if $\Delta(s)=1$ for all $s$ in $g$,

$$
\inf \int_{a}\left|\sum_{1}^{N} c_{n} f\left(x s_{n}^{-1}\right)\right| d x=\int_{a / g}\left|\int_{g} f(x s) \chi\left(x s^{-1}\right) d s\right| d x^{\prime},
$$

where infimums are taken as in 3.2 .

We need only apply (4.4) to $f_{1}=f \chi^{-1} \in L_{1}(\chi(x) d x)$, which yields

$$
\begin{gathered}
\inf \int_{G}\left|\Sigma c_{n} \delta\left(s_{n}^{-1}\right) f\left(x s_{n}^{-1}\right) \chi\left(x s_{n}^{-1}\right)^{-1}\right| \chi(x) d x \\
=\int_{G / g}\left|\int_{g} f(x s) \chi(x s)^{-1} d s\right| d x^{\prime} .
\end{gathered}
$$

Since $\delta\left(s_{n}^{-1}\right) \chi\left(x s_{n}^{-1}\right)^{-1} \chi(x)=\delta\left(s_{n}^{-1}\right) \chi\left(s_{n}\right)=\Delta\left(s_{n}^{-1}\right)$ by (4.2), this becomes

$$
\inf \int_{\theta}\left|\sum_{1}^{N} c_{n} \Delta\left(s_{n}^{-1}\right) f\left(x s_{n}^{-1}\right)\right| d x=\int_{G / g}\left|\int_{g} f(x s) \chi(x s)^{-1} d s\right| d x^{\prime},
$$

yielding (4.6) when $\Delta \equiv 1$ on $g$. Noting that we have defined $f_{s}$ by $f_{s}(x)=f(s x)$, we have

$$
\begin{aligned}
\left(\left(f^{*}\right)_{s}\right)^{*}(x) & =\overline{\left(f^{*}\right)_{s}\left(x^{-1}\right)} \Delta\left(x^{-1}\right)=\overline{f^{*}\left(s x^{-1}\right)} \Delta\left(x^{-1}\right) \\
& =f\left(x s^{-1}\right) \Delta\left(x s^{-1}\right) \Delta\left(x^{-1}\right)=\Delta\left(s^{-1}\right) f\left(x s^{-1}\right),
\end{aligned}
$$

so the left side of (4.7) is

$$
\inf \left\|\Sigma c_{n}\left(\left(f^{*}\right)_{s_{n}}\right)^{*}\right\|_{1}=\inf \left\|\Sigma c_{n}\left(f^{*}\right)_{s_{n}}\right\|_{1},
$$

since ${ }^{*}$ is an isometry, and (4.7) may be rewritten as

$$
\inf \int_{G}\left|\Sigma c_{n} f^{*}\left(s_{n} x\right)\right| d x=\int_{\theta / g}\left|\int_{g} f(x s) \chi\left(x s^{-1}\right) d s\right| d x^{\prime}
$$

which reduces to (4.5) when $f$ is replaced by $f^{*}$.

Finally we should note that there is also a considerable simplification in the technical details surrounding 3.2 in the above situation. Indeed 3.3 then follows from the fact that elements of $L_{1}(G)$ translate continuously, while the use of Lemma 3.1 can easily be avoided by using an approximate identity $\{u\}$. For with $\psi^{s}=\psi$ in $L_{\infty}(G), s \in g$, and $c \leqq|\langle f, \psi\rangle|=$ $\left|\int_{\theta} f(x) \chi(x) \psi(x) d x\right|$, the fact that $f_{0}=f \chi \in L_{1}(G)$ implies $c-\varepsilon \leqq$ $\left|\int_{\theta} u * f_{0}(x) \psi(x) d x\right|$ for some $u$, and this reduces easily to

$$
c-\varepsilon \leqq\left|\left\langle f, \psi_{u}\right\rangle\right|
$$

where $\psi_{u}(x)=\int_{a} u(y) \psi(y x) d y$. But $\psi_{u}$ is a continuous function (bounded by 1 ) which satisfies $\psi_{u}^{s}=\psi_{u}$ in $L_{\infty}(G), s$ in $g$, hence must be constant on cosets mod $g$, which is clearly all that is needed. 
5. We conclude by noting the rather simple analogue of 4.1 which holds for $L_{p}(G), 1<p<\infty$, when $G / g$ carries an invariant measure (corresponding to $\chi \equiv 1$, so that $\Delta(s)=\delta(s), s$ in $g$, by (4.2)). Suppose $S$ is now any generating subsemigroup of $g$. Let $C_{f}^{l}$ (resp. $C_{f}^{r}$ ) denote the closed convex hull of the left (resp. right) translates of $f \in L_{p}(G)$ by elements of $S$. Then either $0 \in C_{f}^{l}$ for every $f$ in $L_{p}(G)$, or $g$ is compact and

$$
\begin{aligned}
\inf \left\{\|h\|_{p}: h \in C_{f}^{r}\right\} & =\left(\int_{G / g}\left|\int_{g} f(x s) d s\right|^{p} d x^{\prime}\right)^{1 / p}, \\
\inf \left\{\|h\|_{p}: h \in C_{f}^{l}\right\} & =\left(\int_{G / g}\left|\int_{g} f^{*}(x s) d s\right|^{p} d x^{\prime}\right)^{1 / p},
\end{aligned}
$$

where $f(x)=\overline{f\left(x^{-1}\right)} \Delta\left(x^{-1}\right)^{(1 / p)}$ (the positive root), and we normalize the Haar measure of $g$ to be 1. (If $g$ is unimodular and noncompact, $0 \in C_{f}^{r}$ for all $f$ in $L^{p}(G)$ as well.)

Indeed, since $L_{p}(G)$ is reflexive and $C_{f}^{l}$ is bounded, $C_{f}^{l}$ has an element $h$ of least norm, unique since the unit ball of $L_{p}(G)$ is strictly convex. But $h_{s} \in C_{f}^{l}$ and $\left\|h_{s}\right\|_{p}=\|h\|_{p}, s$ in $S$, so

$$
h_{s}=h
$$

for all $s$ in $S$, hence for all $s$ in $g$ (since elements of $L_{p}(G)$ translate continuously).

But if $0 \notin C_{f}^{l}$ then $h \neq 0$ and clearly (5.3) cannot hold for all $s$ in $g$ unless $g$ is compact. (A similar argument applies if $g$ is unimodular and $0 \notin C_{f}^{r}$.)

Now with $g$ compact (and so unimodular), the unique element $h$ of least norm in $C_{f}^{r}$ satisfies $h^{s}=h, s$ in $g$, and so with $\mu$ the normalized Haar measure of $g, h * \mu=\int_{g} h^{s} d s=h$, and $h$ is (the equivalence class of) a function constant on cosets $x g$; hence $\|h\|_{p}=\left(\int_{\theta / g} \int_{g}|h(x s)|^{p} d s d x^{\prime}\right)^{1 / p}$ coincides with

$$
\left(\int_{a / \theta}\left|\int_{g} h(x s) d s\right|^{p} d x^{\prime}\right)^{1 / p} .
$$

Since $\Delta(s)=\delta(s)=1$ for $s$ in $g,(5.4)$ coincides with the right side of (5.1) (for any $h$ in $C_{f}^{r}$ ), yielding (5.1).

To obtain (5.2) one employs the (isometric) involution $f \rightarrow f^{*}$ of $L_{p}(G)$, as earlier, noting that $f^{* *}=f_{\mathrm{s}^{-1}}$.

\section{REFERENCES}

1. N. Bourbaki, Espaces Vectoriels Topologiques, Paris, 1953.

2. M. M. Day, Normed linear spaces, Ergebnisse der Math, 21 (1958). 
3. — Amenable semigroups, Illinois J. of Math, 1 (1958), 509-543.

4. - Fixed-point theorems for compact convex sets, Illinois J. of Math, 5 (1961), 585-590.

5. N. Dunford, and J. Schwartz, Linear Operators I, New York, 1958.

6. K. Jacobs, Neuere Methoden und Ergebnisse der Ergodentheorie, Ergeb. der Math., vol. 29, Berlin, 1960.

7. H. Reiter, Contributions to harmonic analysis, Acta Math., 96 (1956), 253-263.

8. - Ü̈ber $L^{1-R a ̈ u m e ~ a u f ~ G r u p p e n ~} I, I I$, Monatshefte für Math., 58 (1954), 73-76, $172-180$

9 . The convex hull of translates of a function in $L^{1}$, J. London Math. Soc., 35 (1960), 5-16.

10. S. Świerczkowski, Integrals on quotient spaces, Colloq. Math., 8 (1961), 107-114.

11. A. Weil, L'intégration dans les groupes topologiques et ses applications, $2^{e}$ éd., Paris, 1953.

INSTITUTE FOR ADVANCED STUDY

UNIVERSITY OF WASHINGTON 



\section{PACIFIC JOURNAL OF MATHEMATICS}

\section{EDITORS}

\section{RalPh S. Phillips}

Stanford University

Stanford, California

M. G. Arsove

University of Washington

Seattle 5, Washington
J. Dugundji

University of Southern California Los Angeles 7, California

Lowell J. Paige

University of California

Los Angeles 24, California

\section{ASSOCIATE EDITORS}
E. F. BECKENBACH
D. DERRY
H. L. ROYDEN
E. G. STRAUS
T. M. CHERRY
M. OHTSUKA
E. SPANIER
F. WOLF

\section{SUPPORTING INSTITUTIONS}

\author{
UNIVERSITY OF BRITISH COLUMBIA \\ CALIFORNIA INSTITUTE OF TECHNOLOGY \\ UNIVERSITY OF CALIFORNIA \\ MONTANA STATE UNIVERSITY \\ UNIVERSITY OF NEVADA \\ NEW MEXICO STATE UNIVERSITY \\ OREGON STATE UNIVERSITY \\ UNIVERSITY OF OREGON \\ OSAKA UNIVERSITY \\ UNIVERSITY OF SOUTHERN CALIFORNIA
}

STANFORD UNIVERSITY

UNIVERSITY OF TOKYO

UNIVERSITY OF UTAH

WASHINGTON STATE UNIVERSITY

UNIVERSITY OF WASHINGTON

AMERICAN MATHEMATICAL SOCIETY

CALIFORNIA RESEARCH CORPORATION SPACE TECHNOLOGY LABORATORIES

NAVAL ORDNANCE TEST STATION

Printed in Japan by International Academic Printing Co., Ltd., Tokyo Japan 


\section{Pacific Journal of Mathematics}

\section{Vol. 13, No. 1 \\ March, 1963}

Frantz Woodrow Ashley, Jr., A cone of super-(L) functions............. 1

Earl Robert Berkson, Some metrics on the subspaces of a Banach space....

Felix Earl Browder and Walter Strauss, Scattering for non-linear wave

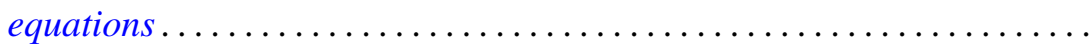

Edmond Darrell Cashwell and C. J. Everett, Formal power series ..........

Frank Sydney Cater, Continuous linear functionals on certain topological

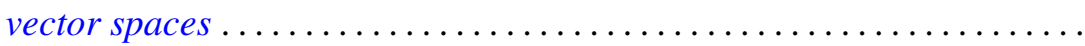

John Douglas Dixon, General group extensions ....................

Robert Pertsch Gilbert, On harmonic functions of four variables with

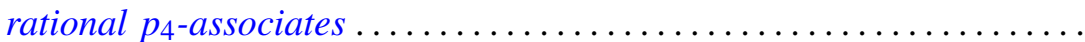

Irving Leonard Glicksberg, On convex hulls of translates ..............

Simon Hellerstein, On a class of meromorphic functions with deficient zeros

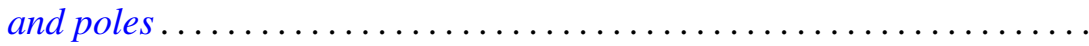

Donald William Kahn, Secondary cohomology operations which extend the

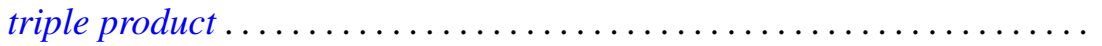

G. K. Leaf, A spectral theory for a class of linear operators .............

R. Sherman Lehman, Algebraic properties of the composition of solutions of partial differential equations ........................... 157

Joseph Lehner, On the generation of discontinuous groups ............. 169

S. P. Lloyd, On certain projections in spaces of continuous functions ...... 171 Fumi-Yuki Maeda, Generalized spectral operators on locally convex spaces ..................................

Donald Vern Meyer, $E^{3}$ modulo a 3-cell

William H. Mills, An application of linear programming to permutation groups.

Richard Scott Pierce, Centers of purity in abelian groups

Christian Pommerenke, On meromorphic starlike functions ...

Zalman Rubinstein, Analytic methods in the study of zeros of

polynomials...

B. N. Sahney, On the Nörlund summability of Fourier series

Tôru Saitô, Regular elements in an ordered semigroup . .

Lee Meyers Sonneborn, Level sets on spheres...........

Charles Andrew Swanson, Asymptotic estimates for limit point

problems .

Lucien Waelbroeck, On the analytic spectrum of Arens . .

Alvin (Murray) White, Singularities of a harmonic function of three

variables given by its series development .............

Kōichi Yamamoto, Decomposition fields of difference sets ...

Chung-Tao Yang, On the action of $\mathrm{SO}(3)$ on a cohomology manifold... 